\section{Hypofractionated radiation therapy versus conventionally fractionated radiation therapy for early-stage breast cancer: how do we choose?}
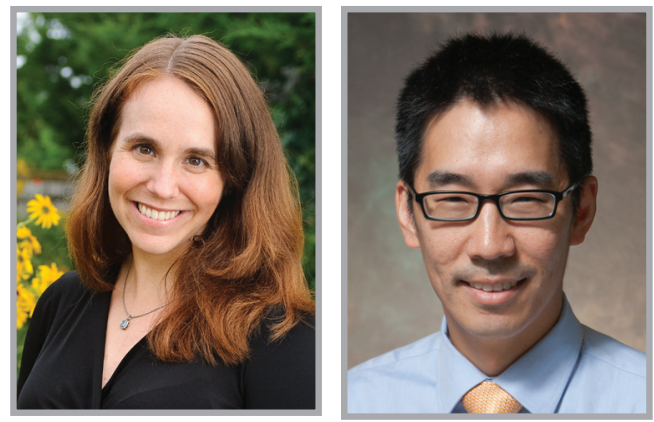

\author{
“Hypofractionated \\ radiotherapy should be \\ routinely offered to women \\ who desire breast conserving \\ therapy with invasive, \\ node-negative breast cancer, \\ regardless of the grade of \\ invasive disease."
}

Suzanne B Evans ${ }^{1,2} \&$ James B Yu ${ }^{* 1,2}$

Hypofractionated whole-breast irradiation (HF-WBI), meaning the use of daily doses of radiation greater than $2.0 \mathrm{~Gy}$, is an extensively studied method of delivering radiotherapy as a component of breast conserving care after lumpectomy for early-stage breast cancer. Since the longterm results of seminal trials [1], use of hypofractionated radiotherapy has been increasing worldwide. However, many radiation oncologists still resist the routine adoption of hypofractionated radiotherapy. Two overarching questions arise: In which patients is it appropriate to adopt hypofractionated radiation? What are the societal benefits of hypofractionated radiation?

In which patients is it appropriate to offer hypofractionated radiotherapy? Hypofractionated radiation therapy for breast cancer is supported by several large randomized clinical trials for treatment of early invasive breast cancer [1,2]. Patients within these trials had T1-T2 N0 disease, predominantly, and only approximately $1 \%$ of the study population had the supraclavicular fossa treated. Strict homogeneity criteria were applied in some manner for all trials. Although there was initial caution expressed by the American Society of Therapeutic Radiology and Oncology (ASTRO) practice guidelines, there is now data to support the safety of the use of HF-WBI in patients having received chemotherapy [3]. Clinicians should also feel comfortable applying HF-WBI in patients with pure ductal carcinoma in situ, as there are several large retrospective analyses demonstrating its efficacy [4].

Efficacy is clear, however, how do the side effects compare? Acute skin toxicity has been favorably compared and so should not be considered a barrier to HF-WBI treatment compared with standard fractionation $[5,6]$. Late skin toxicity has been evaluated by ultrasound, and was found to be grade 1 in over $80 \%$ of the cases [7]. A report on health-related quality of life favored HF-WBI [8], which exhibited faster recoveries to normal with the shorter regimen. The UK START trials included an analysis of cardiovascular events which

\section{KEYWORDS}

- breast cancer $\bullet$ breast conserving therapy $\bullet$ hypofractionated whole-breast radiotherapy - hypofractionation • radiotherapy

\footnotetext{
“Hypofractionated whole-breast irradiation ... is an extensively studied method of delivering radiotherapy as a component of breast conserving care after lumpectomy for early-stage breast cancer."
} 


\section{"Physicians should not be held back in offering hypofractionated radiotherapy due to concerns about toxicity or cosmesis."}

were not different between conventional and HF-WBI, and this is supported elsewhere [9,10].

However, there is limited evidence with short follow-up for the use of HF-WBI post mastectomy [11]. The limited numbers of patients and the potentially long time to development of brachial plexopathy have made many clinicians appropriately hesitant about the use of HF regimens in setting of regional nodal irradiation. Additionally, it is undefined what the appropriate homogeneity constraints should be in the regional nodal areas. Likewise, this regimen remains essentially untested in young women, in those with implants or autologous reconstruction, in combination with bolus, or with advanced local disease. The Yale experience has not included women with these characteristics, although a clinical trial is eagerly awaited for these subpopulations.

Therefore, at our institution, we offer and recommend HF-WBI to all women requiring breast only radiation provided the radiation treatment plan can achieve a global max hot spot of $107 \%$ (with infrequent variations to $109 \%$ in selected cases), in women with their native breast intact over the age of 40 , regardless of the use of chemotherapy with success.

\section{What are the societal benefits of hypofractionated radiation?}

Hypofractionated breast radiotherapy, compared with standard fractionation, allows for a more abbreviated length of treatment. In the USA fee-for-service environment, where each treatment is individually reimbursed to the treating facility (and each week of management of radiation treatment is reimbursed to the physician), a reduced length of treatment results in a proportionally reduced cost. Therefore, in addition to being more convenient to the patient, the overall cost of radiation treatment is reduced. Notably the cost of treatment preparation is proportionally larger when the cost of the actual delivery is reduced. This potential cost reduction has been replicated in other healthcare systems [12]. For example, in a Belgian study, the cost of hypofractionated radiation was much lower (by $60 \%)$ in comparison to standard fractionated treatment [13].

The potential benefit to the patient, in terms of cost and convenience, will only be worthwhile if there is no difference in cancer outcomes or quality of life after treatment. As noted above, at least equivalent outcomes after hypofractionated breast radiation has now been proven in randomized trials with long-term follow-up. Further data are emerging or has matured for other disease sites. For example, for prostate cancer, the Fox Chase Cancer Center randomized trial showed no significant differences between hypofractionated radiation and standardly fractionated radiotherapy [14]. Notably, this trial was not powered to detect noninferiority of hypofractionated radiation, but rather rejected the hypothesis that hypofractionated radiation was superior to standard fractionation. Therefore, though hypofractionated prostate radiotherapy appears promising, some investigators and physicians desire more proof before wholeheartedly embracing hypofractionation for prostate cancer. For early-stage lung cancer, radiosurgery (extremely hypofractionated radiotherapy) is the standard of care given overwhelming evidence indicating its superiority to conventionally fractionated treatment. However, for locally advanced lung cancer requiring larger lung fields and potentially treatment of regional lymph nodes, the utility of hypofractionated therapy is the subject of active investigation [15].

Regardless of the impact and evidence of hypofractionation for other disease sites, for breast cancer, the data have spoken. However, despite significant data and support for hypofractionated breast cancer, it is not yet being used extensively in the USA. In comparison, roughly $70 \%$ of eligible women undergo hypofractionated radiotherapy for breast cancer [16]. Our recent analysis shows that hypofractionated radiation for breast cancer has increased from $5.4 \%$ in 2004 to $22.8 \%$ in 2011 in the USA [17]. Given that much of the concern regarding longterm toxicity from hypofractionated radiation have been addressed with the publication of the above clinical trials, it is likely that hypofractionated breast irradiation will likely increase. Furthermore, hypofractionated breast radiotherapy has arguably become the preferred standard of care in eligible patients [18]. In conjunction with this realization, ASTRO supports the routine use of hypofractionated radiotherapy in its 'Choosing Wisely' recommendation.

\section{Conclusion}

Hypofractionated radiotherapy should be routinely offered to women who desire breast conserving therapy with invasive, node-negative breast cancer, regardless of the grade of invasive disease. Physicians should not be held back in 
offering hypofractionated radiotherapy due to concerns about toxicity or cosmesis. Further national adoption of hypofractionated radiotherapy can potentially save the healthcare system billions of dollars over the coming years, as well as improve the convenience of breast conserving radiotherapy.

At our institution, hypofractionated breast radiation has become the standard of care for eligible women. Though this has reduced the potential revenue for our department and our physician practice, we believe it is the right thing to do for our patients and as members of a fiscally taxed health system.

\section{Financial \& competing interests disclosure}

$J B Y u$ and $S B$ Evans receive research support from 21st Century Oncology. The authors have no other relevant affliations or financial involvement with any organization or entity with a financial interest in or financial conflict with the subject matter or materials discussed in the manuscript apart from those disclosed.

No writing assistance was utilized in the production of this manuscript.

\section{References}

1 Whelan TJ, Pignol JP, Levine MN et al. Long-term results of hypofractionated radiation therapy for breast cancer. $N$. Engl. J. Med. 362(6), 513-520 (2010).

2 Haviland JS, Owen JR, Dewar JA et al. The UK Standardisation of Breast Radiotherapy (START) trials of radiotherapy hypofractionation for treatment of early breast cancer: 10-year follow-up results of two randomised controlled trials. Lancet Oncol. 14(11), 1086-1094 (2013).

3 Kouloulias V, Zygogianni A, Kypraiou E et al. Adjuvant chemotherapy and acute toxicity in hypofractionated radiotherapy for early breast cancer. World J. Clin. Cases 2(11), 705-710 (2014).

4 Lalani N, Paszat L, Sutradhar R et al. Long-term outcomes of hypofractionation versus conventional radiation therapy after breast-conserving surgery for ductal carcinoma in situ of the breast. Int. J. Radiat. Oncol. Biol. Phys. 90(5), 1017-1024 (2014).

5 Hopwood P, Sumo G, Mills J et al. The course of anxiety and depression over 5 years of follow-up and risk factors in women with early breast cancer: results from the UK Standardisation of Radiotherapy Trials (START). Breast 19(2), 84-91 (2010).

6 Tortorelli G, Di Murro L, Barbarino R et al. Standard or hypofractionated radiotherapy in the postoperative treatment of breast cancer: a retrospective analysis of acute skin toxicity and dose inhomogeneities. BMC Cancer 13 , 230 (2013).

7 Landoni V, Giordano C, Marsella A et al. Evidence from a breast cancer hypofractionated schedule: late skin toxicity assessed by ultrasound. J. Exp. Clin. Cancer Res. 32, 80 (2013).

8 Versmessen H, Vinh-Hung V, Van Parijs H et al. Health-related quality of life in survivors of stage I-II breast cancer: randomized trial of post-operative conventional radiotherapy and hypofractionated tomotherapy. BMC Cancer 12, 495 (2012).

9 Chan EK, Woods R, Virani S et al. Long-term mortality from cardiac causes after adjuvant hypofractionated vs. conventional radiotherapy for localized left-sided breast cancer. Radiother. Oncol. 114(1), 73-78 (2015).

10 Chan EK, Woods R, McBride ML et al. Adjuvant hypofractionated versus conventional whole breast radiation therapy for early-stage breast cancer: long-term hospital-related morbidity from cardiac causes. Int. J. Radiat. Oncol. Biol. Phys. 88(4), 786-792 (2014).

11 Ko DH, Norriss A, Harrington CR et al. Hypofractionated radiation treatment following mastectomy in early breast cancer: the Christchurch experience. J. Med. Imaging Radiat. Oncol. 59(2), 243-247 (2015).
12 Dwyer P, Hickey B, Burmeister E, Burmeister B. Hypofractionated whole-breast radiotherapy: impact on departmental waiting times and cost. J. Med. Imaging Radiat. Oncol. 54(3), 229-234 (2010).

13 Lievens Y. Hypofractionated breast radiotherapy: financial and economic consequences. Breast 19(3), 192-197 (2010).

14 Pollack A, Walker G, Horwitz EM et al. Randomized trial of hypofractionated external-beam radiotherapy for prostate cancer. J. Clin. Oncol. 31(31), 3860-3868 (2013).

15 Cannon DM, Mehta MP, Adkinson JB et al. Dose-limiting toxicity after hypofractionated dose-escalated radiotherapy in non-small-cell lung cancer. J. Clin. Oncol. 31(34), 4343-4348 (2013).

16 Ashworth A, Kong W, Whelan T, Mackillop WJ. A population-based study of the fractionation of postlumpectomy breast radiation therapy. Int. J. Radiat. Oncol. Biol. Phys. 86(1), 51-57 (2013).

17 Wang EH, Mougalian SS, Soulos PR. Adoption of hypofractionated whole-breast irradiation for early-stage breast cancer: a national cancer data base analysis. Int. J. Radiat. Oncol. Biol. Phys. 90(5), 993-1000 (2014).

18 Haffty BG, Buchholz TA. Hypofractionated breast radiation: preferred standard of care? Lancet Oncol. 14(11), 1032-1034 (2013). 